\title{
Optimal Booking Limits in the Presence of Strategic Consumer Behavior
}

\author{
John G. Wilson, Chris K. Anderson and Sang-Won Kim \\ University of Western Ontario
}

\begin{abstract}
We consider a two-period airline yield management problem where customers may act strategically. Specifically, we allow for the possibility that a customer may decide to defer purchase in the hope that a ticket cheaper than those currently on offer will become available. We also allow for the possibility that some customers will buy a more expensive ticket if the cheaper tickets are not available. We show how to find optimal booking limits in the presence of such strategic customer behavior. We also explicitly incorporate the fact that, once a booking limit has been reached, demand distributions are now censored distributions.
\end{abstract}

\section{Introduction}

During any given period, an airline has an inventory of seats available for sale in different fare classes. Using purchase patterns and projections of future sales in the various fare classes, an airline will typically move inventory between classes in order to maximize expected revenue. Littlewood (1972) considered a one-period model with two fare classes. Belobaba (1989) developed the expected marginal seat revenue (EMSR) approach which extended Littlewood's one-period model to the case of multiple fares. Pfeifer (1989), Bodily and Weatherford (1995), Belobaba and Weatherford (1996) and Weatherford et al. (1993) analyze single-period models where a customer may buy a more expensive ticket if cheaper ones are not available. Sen and Zhang (1999) analyze a single-period model with two fare classes where it is assumed that a certain fraction of customers will purchase the more expensive item if cheaper ones are not available. For this case, they provide a procedure for finding the booking limit (the limit on the number of items that can be sold at a cheaper price) that maximizes the company's expected revenue.

An airline, of course, has many opportunities for the reallocation of seats to fare classes. Singleperiod models are certainly valuable and provide insight. However, ideally, models that take advantage of the fact that allocations can be made during different periods are desirable. Belobaba's EMSR model 
can be repeatedly applied to allow for multiple periods. The early approaches to deal with the flexibility in resetting booking limits simply reapplied the static one- period models, in what has been termed advanced static approaches (Weatherford and Bodily, 1992). Robinson (1995) develops an optimal multi-period approach using Monte Carlo integration. Robinson assumes that customers arrive in sequential fare order, low to high, in each of the periods with demands across periods assumed to be independent. This approach allows for the realization that posted fares are not monotonic as currently closed cheaper fares are often available in the future. Both the re-application of single-period approaches or multi-period approaches like Robinson's assume that demands in each fare class are independent and that customers do not behave strategically.

A separate stream of research that does not incorporate diversion has used dynamic programming formulations to develop optimal dynamic allocations. Hersh and Ladany (1978) and Ladany and Bedi (1977) use dynamic programming in allocating seats on a two-segment flight. Gerchak et al. (1985) develop a model equivalent to a two fare airline model, with Lee and Hersh (1993) developing extensions for more than two fares. Lee and Hersh break the decision horizon into numerous stages such that only one request is received per period, enabling a finite state space. The work of Lee and Hersh, in particular their use of numerous short periods, has formed the basis of considerable further research.

Anderson and Wilson (2003) investigated the effect on revenue when customers behave strategically (defer purchase in the hope that a cheaper ticket will become available) but booking limits are set assuming that demands in each period for each fare class are independent. In this paper, we start to address the problem of how to modify the booking limits to make allowance for such strategic behavior. Following Robinson (1995), we assume customers arrive in fare order within a period. We allow for diversion as discussed by Pfeifer (1989) and include the realization that demands between periods (and fare classes) are potentially dependent as a function of diversion and potential strategic behavior.

\section{Optimal Two-Period Model}

We will analyze the situation where there are two periods and two fare classes. As in Robinson (1995), we assume that low fare customers arrive before high fare customers in each period. However, we now extend that approach by assuming that a fraction of customers in the first period who cannot get a low fare will purchase the high fare and a fraction will wait until the second period in the hope that a low fare will become available. In the second and final period we assume that a fraction of customers 
who cannot get a low fare will buy a higher fare. Optimal results for the single period, two fare class problem are relatively recent (Sen and Zhang, 1999) and will provide the foundation for solving the twoperiod problem. Assume that in period $i$, a fraction $d_{i}(i=1,2)$ of customers will purchase the more expensive ticket if the cheaper one is not available. We allow for consumer strategic behavior by assuming that a fraction $w$ will wait until period 2 if a cheap fare is not available in period 1 . The capacity at the beginning of period 1 will be denoted $C$ and the capacity at the beginning of period 2 denoted $c$, with $\mathrm{C}-\mathrm{c}>0$ the seats sold in period 1 . There are two fare classes with revenues of $r_{1}$ and $r_{2}$, where $r_{1}<r_{2}$. For $\mathrm{i}, j \in 1,2$, the demand for fare class $\mathrm{j}$ in period $\mathrm{i}$ will be denoted by $D_{i, j}$. The corresponding density function will be denoted by $f_{i, j}(\cdot)$.

In the second period the optimal booking limit is a function of the remaining capacity and of the first period booking limit if that limit was reached. The procedure for finding the optimal booking limits for the second period is outlined in Section 2.1. The distribution of capacity at the beginning of the second period is derived in Section 2.2. The procedure for finding the optimal booking limit in the first period is provided in Section 2.3.

\section{Booking Limits and Expected Returns for Period 2}

Suppose the booking limit for period 1 is I1. Then, given this I1, the optimal booking limit in period 2 is a function of the capacity at the end of period 1 and whether or not lower fare demand in the first period exceeded the booking limit. Let $I_{2}(c)$ denote the booking limit in period 2 if the capacity at the end of period 1 is $\mathrm{c}$ and the booking limit I1 was not reached in period 1 . In this case, there are no customers who wait until period 2 in the hope of obtaining a cheaper fare as plenty of these fares were available in period 1 . Let $I_{1}\left(c, I_{1}\right)$ denote the optimal booking limit in period 2 if all $I_{1}$ of the cheap fares are sold in period 1 and c seats remain going into period 2 . For this case, some of the customers unable to obtain a cheaper fare in period 1 will try again in period 2 . The precise number of such customers is, however, unknown because the number of lower fare customers in period 1 is censored at $I_{1}$.

Consider the case where the booking limit for the cheaper fare is $I_{1}$ in period $1, D_{1,1}$ is less than $I_{1}$ and there is capacity $c$ going into the second period. Then the optimal booking limit, $I_{2}(c)$, for the cheaper fare in period 2 is either $0, \mathrm{c}$ or the value of $\mathrm{I}_{2}$ that satisfies the following equation (rearrange expression (9) of Sen and Zhang, 1999): 
(1)

$$
\begin{aligned}
& \left(r_{1}-r_{2}\right)+\left(r_{2}-r_{1}\right) P\left[D_{2,1}<l_{2}\right]-r_{2}\left(1-d_{2}\right) P\left[D_{2,2}<c-l_{2}\right] \\
& \quad+r_{2}\left(1-d_{2}\right) \int_{0}^{c-l_{2}} P\left[D_{2,1}<\frac{c-l_{2}\left(1-d_{2}\right)-x}{d_{2}}\right] f_{2,2}(x) \mathrm{d} x=0 .
\end{aligned}
$$

Now, suppose that $I 1$ is the booking limit in period 1 , that all of these seats are sold and that there are $c$ unsold seats going into the second period. In this situation, all that is known about the demand for the cheaper seats in period 1 is that it was at least $I_{1}$. The conditional distribution function for the number of customers, $Y$, who will wait until the second period is

(2)

$$
=P\left[l_{1} \leqslant D_{1,1} \leqslant \frac{y}{w}+l_{1}\right] \frac{1}{P\left[D_{1,1} \geqslant l_{1}\right]} .
$$

Differentiate the above to see that the density function for $Y$ is given by

(3)

$$
f_{Y}(y)=\frac{f_{1,1}\left(l_{1}+\frac{y}{w}\right)}{w P\left[D_{1,1} \geqslant l_{1}\right]} \quad \text { for } y \geqslant 0 .
$$

The demand for cheaper fares in period 2 is $D_{2,1}$ plus the demand from those customers who wait for period 2. Obtaining the optimal booking limit for the second period now becomes a one- period problem and can be found from (1) by replacing D2,1 with D2,11Y. Denote this random variable by $D_{2,1}\left(I_{1}\right)$. Replace $P\left[D_{2,1}<_{2}\right]$ in (1) with 
(4)

$$
\begin{aligned}
P\left[D_{2,1}\left(l_{1}\right)<l_{2}\right] & =P\left[D_{2,1}+Y<l_{2}\right] \\
& =\int_{0}^{l_{2}} P\left[D_{2,1}<l_{2}-y\right] f_{Y}(y) \mathrm{d} y \\
& =\frac{\int_{0}^{l_{2}} P\left[D_{2,1}<l_{2}-y\right] f_{1,1}\left(l_{1}+{ }_{w}^{y}\right) \mathrm{d} y}{w P\left[D_{1,1} \geqslant l_{1}\right]} .
\end{aligned}
$$

(5)

Similarly, replace $P\left[D_{2,1}<\frac{c-l_{2}\left(1-d_{1}\right)-x}{d_{2}}\right]$ in (1) with

$$
P\left[D_{2,1}\left(l_{1}\right)<\frac{c-l_{2}\left(1-d_{2}\right)-x}{d_{2}}\right] .
$$

Now the optimal booking limit in period $2, I_{2}\left(c, I_{1}\right)$, is a function of both $I_{1}$ and the number of available seats. Use (4) and (5) in (1) to see that $I_{2}\left(c, I_{1}\right)$ is either $0, c$ or the value of $I_{2}$ that satisfies the following equation:

(6)

$$
\begin{aligned}
& w P\left[D_{1,1} \geqslant l_{1}\right]\left(r_{1}-r_{2}\right)+\left(r_{2}-r_{1}\right) \int_{0}^{h_{2}} P\left[D_{2,1}<l_{2}-y\right] f_{1,1}\left(l_{1}+\frac{y}{w}\right) \mathrm{d} y \\
& -w P\left[D_{1,1} \leqslant l_{1}\right] r_{2}\left(1-d_{2}\right) P\left[D_{2,2}<c-l_{2}\right] \\
& +r_{2}\left(1-d_{2}\right) \int_{0}^{c-l_{2}} \int_{0}^{\frac{s,\left(1-y_{2}\right)-x}{d_{2}}} P\left[D_{2,1}<\frac{c-l_{2}\left(1-d_{2} y\right)}{y}\right] f_{1,1}\left(l_{1}+y\right) \mathrm{d} y f_{2,2}(x) \mathrm{d} x=0 .
\end{aligned}
$$

If the capacity going into the second period is $\mathrm{c}$ and the booking limit is not reached in period 1 , then the expected revenue for the second period can be written as

(7)

$r_{1} E\left[\min \left\{D_{2,1}, l_{2}(c)\right\}\right]+$

$r_{2} E\left[\min \left\{\max \left\{c-l_{2}(c), c-D_{2,1}\right\}, D_{2,2}+d_{2}\left(D_{2,1}-l_{2}(c)\right)^{+}\right\}\right]$.

If the capacity is $\mathrm{c}$ going into the second period and the booking limit I 1 is reached in period 1 , then the expected return in the second period is given by (7) with $D_{2,1}$ and $I_{2}(c)$ replaced by $D_{2,1}\left(I_{1}\right)$ and $\mathrm{I}_{2}\left(c, \mathrm{I}_{1}\right)$, respectively. The above is straightforward to calculate and explicit formulas can be found in Sen and Zhang (1999). 


\section{Example}

2.1.1. Example Assume that $40 \%$ of customers are willing to wait from period to period for cheaper fares, and $10 \%$ of customers are willing to buy more expensive fares if cheaper ones are sold out. Plane capacity is 50 with demand being normally distributed with $\mu=15$ and $\sigma=3$ for each fare class in each period, i.e. total demand has $\mu=60$ and $\sigma=6$.

Figure 1 shows optimal period 2 booking limits as a function of remaining capacity and first period booking limits when first period demand met the booking limit. Figure 2 shows the expected revenues under the same setting. As expected, revenues and booking limits increase as more capacity is available. As $I_{1}$ increases, fewer customers are expected to wait until the second period. Consequently, the pool of potential diverters to the more expensive fare becomes smaller which means the optimal booking limit in period 2 increases. 


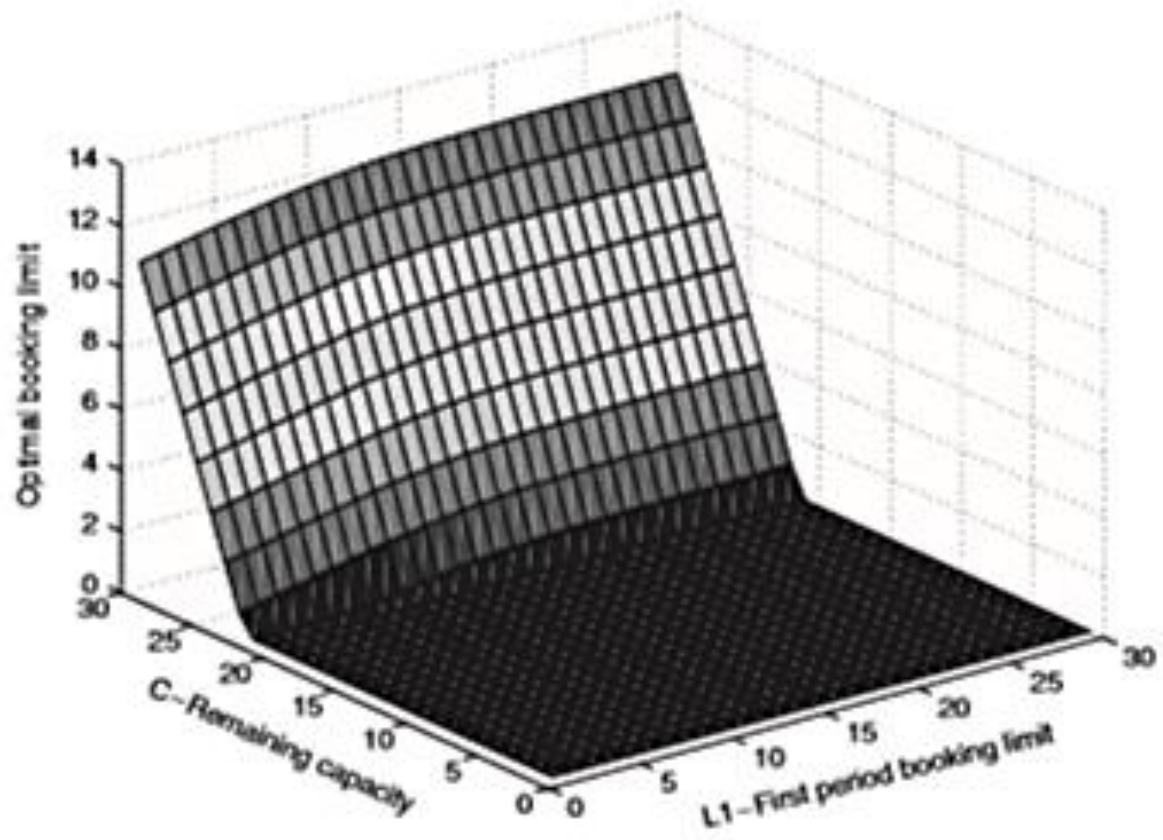

Fig. 1. Period 2 optimal booking limits.

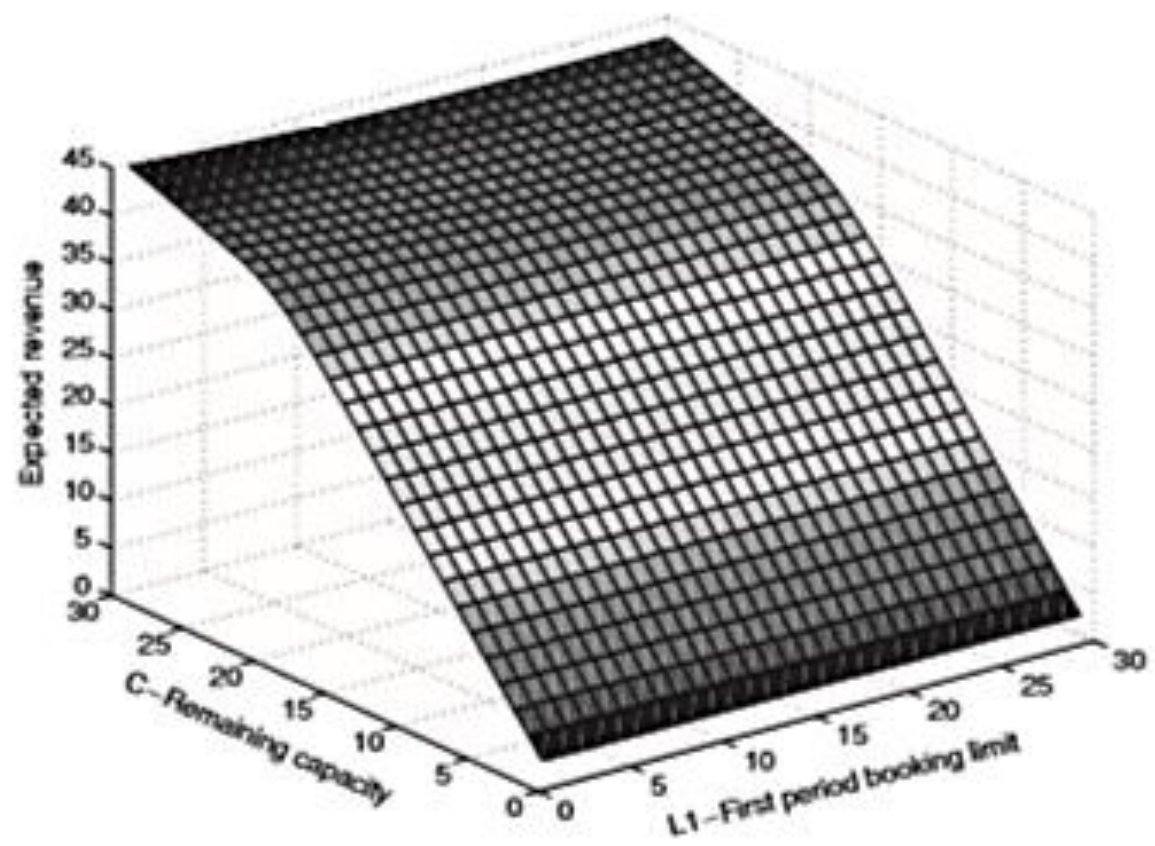

Fig. 2. Period 2 expectod revenue. 


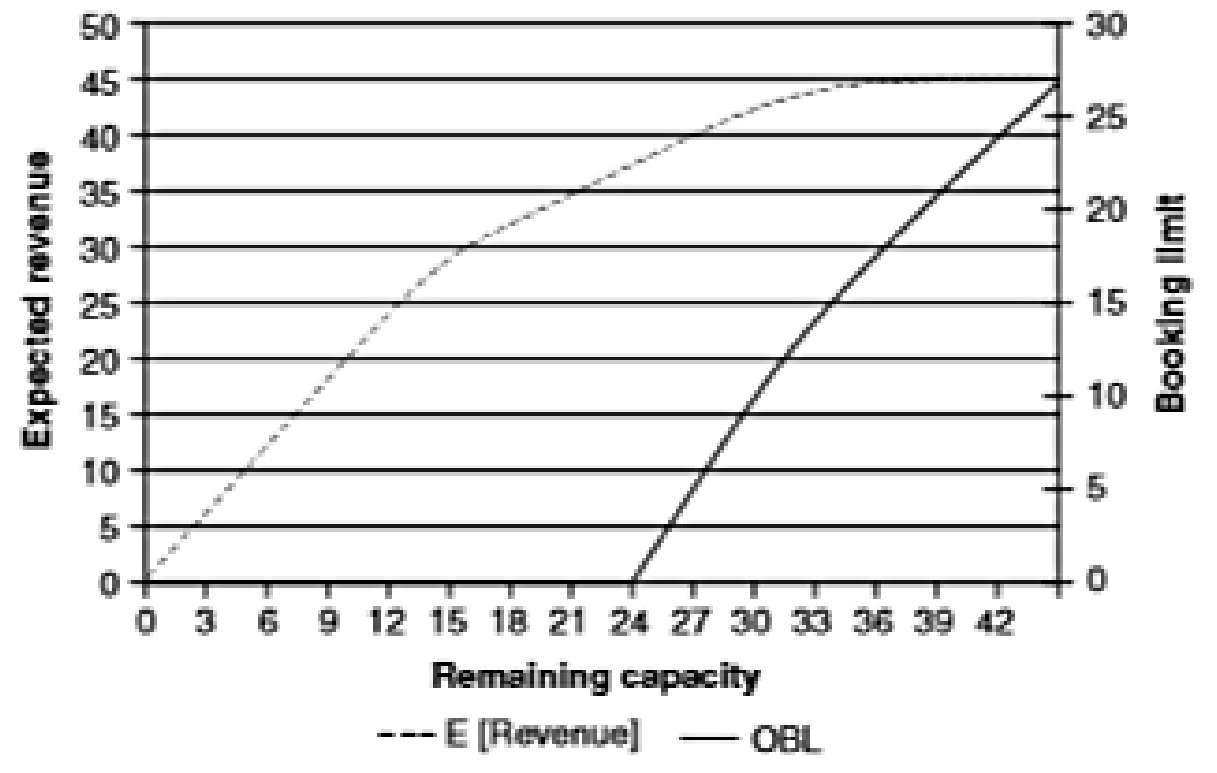

Fig. 3. Period 2 expected revenue and booking limit-uncensored demand.

Figure 3 shows expected revenue and optimal booking limits (OBL) as a function of remaining capacity when first period demand is less than booking limits $\left(D_{1,1}<l_{1}\right)$. Here revenues and booking limits are not a function of $I_{1}$ as no customers need to wait and simply increase with available capacity.

\subsection{Density functions for capacity}

The capacity, $\mathrm{C}\left(\mathrm{I}_{1}\right)$, remaining at the beginning of the second period is given by:

(8)

$$
\begin{aligned}
C\left(l_{1}\right)= & C-\min \left\{D_{1,1}, l_{1}\right\} \\
& -\min \left\{\max \left\{C-l_{1}, C-D_{1,1}\right\}, D_{1,2}+d_{1}\left(D_{1,1}-l_{1}\right)^{+}\right\} .
\end{aligned}
$$

Let $g_{1}\left(c, I_{1}\right)$ denote the density function for $C(I 1)$ conditioned on the assumption that ${ }_{D 1,1}<\left.\right|_{1}$ and $C\left(I_{1}\right)>0$ and let $g_{2}\left(C, I_{1}\right)$ denote the density conditioned on $D_{1,1}>I_{1}$ and $C\left(I_{1}\right)>0$, i.e. 
(9)

$$
g_{1}\left(c, l_{1}\right)=\frac{\frac{\partial}{\partial c} P\left[0<C\left(l_{1}\right) \leqslant c, D_{1,1}<l_{1}\right]}{P\left[D_{1,1}<l_{1}, C\left(l_{1}\right)>0\right]}
$$

and,

(10)

$$
g_{2}\left(c, l_{1}\right)=\frac{\frac{\partial}{\partial c} P\left[0<C\left(l_{1}\right) \leqslant c, D_{1,1} \geqslant l_{1}\right]}{P\left[D_{1,1} \geqslant l_{1}, C\left(l_{1}\right)>0\right]} .
$$

The denominations in (9) and (10) are given by

(11)

$$
P\left[D_{1,1}<l_{1}, C\left(l_{1}\right)>0\right]=\int_{0}^{l_{1}} \int_{0}^{C-x} f_{1,2}(y) \mathrm{d} y f_{1,1}(x) \mathrm{d} x
$$

and

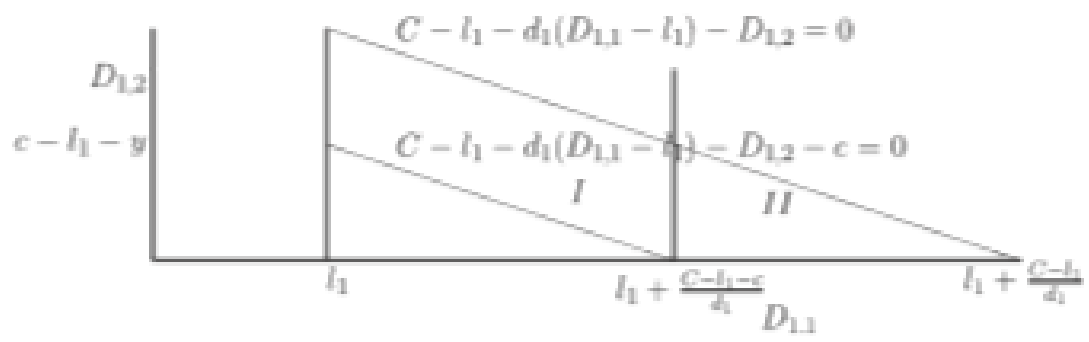

Fig. 4. Find $P\left[0<C\left(I_{1}\right) \leqslant c, D_{1,1} \geqslant I_{1}\right]$ by integrating over regions $\mathrm{I}$ and II.

(12)

$$
P\left[D_{1,1} \geqslant l_{1}, C\left(l_{1}\right)>0\right]=\int_{l_{1}}^{l_{1}+\frac{c h_{1}}{\alpha_{1}}} \int_{0}^{C-l_{1}-d_{1}\left(D_{1,1}-l_{1}\right)} f_{1,2}(y) \mathrm{d} y f_{1,1}(x) \mathrm{d} x,
$$

respectively.

If $D_{1,1}>\left.\right|_{1}$, then there are $C-I_{1}$ seats available at the higher price and seats will only be available for period 2 if $D_{1,2}+d_{1}\left(D_{1,1}-I_{1}\right)$, the demand for the more expensive fares in period 1 , is less than $C-I_{1}$. If this happens, then $C\left(I_{1}\right)<c$ only if $C-I_{1}-D_{1,2}-d_{1}\left(D_{1,1}-I_{1}\right)<c$. Consequently, computing the denominator in (10) requires integration over the regions I and II shown in Fig. 4. 
(13)

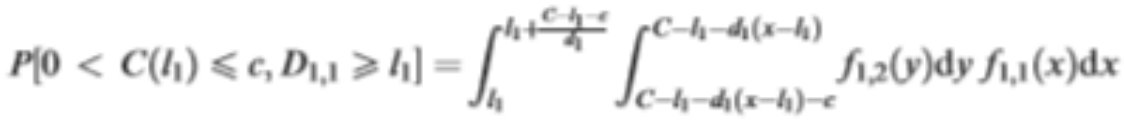

$$
\begin{aligned}
& +\int_{l_{1}+\frac{c_{y}}{h_{1}}}^{h_{1}+\frac{c_{4}}{h_{1}}} f_{1,1}(x) \mathrm{d} x \text {. }
\end{aligned}
$$

Differentiate the above with respect to $c$ and use (10) to see that

$$
g_{2}\left(c, l_{1}\right)=\int_{l_{4}}^{l_{4}+\frac{c y_{1}=}{4}}\left\{f_{1,2}\left(C-l_{1}-d_{1}\left(x-l_{1}\right)-c\right) f_{1,1}(x) \mathrm{d} x\right\} / P\left[D_{1,1} \geqslant l_{1}, C\left(l_{1}\right)>0\right] .
$$

If $D_{1,1}<l_{1}$, then $C\left(l_{1}\right)=C-D_{1,1}-\min \left(C-D_{1,1}, D_{1,2}\right)$ and seats will be available in period 2 only if $C-D_{1,1^{-}}$ $D_{1,2}>0$. In this case, the number of seats will be less than $c$ if $C-D_{1,1}-D_{1,2}<c$. The shape of the region represented by the previous two inequalities and $D_{1,1}<l_{1}$ depends on the value of $c$. From Figs 5 and 6 , there are two cases to be considered in finding the value of $g\left(c, I_{1}\right)$ depending on the value of $c$.

Case 1: $c<C-I_{1}$. From (8) and Fig. 5

(15)

$P\left[C\left(l_{1}\right) \leqslant c, D_{1,1}<l_{1}, C\left(l_{1}\right)>0\right]=\int_{0}^{l_{1}} \int_{C-x-c}^{C-x} f_{1,2}(y) \mathrm{d} y f_{1,1}(x) \mathrm{d} x$.

Differentiate with respect to $c$ and use (9) to see that 
(16)

$g_{1}\left(c, l_{1}\right)=\int_{0}^{l_{1}} f_{1,2}(C-x-c) f_{1,1}(x) \mathrm{d} x / P\left[D_{1,1}<l_{1}, C\left(l_{1}\right)>0\right]$.

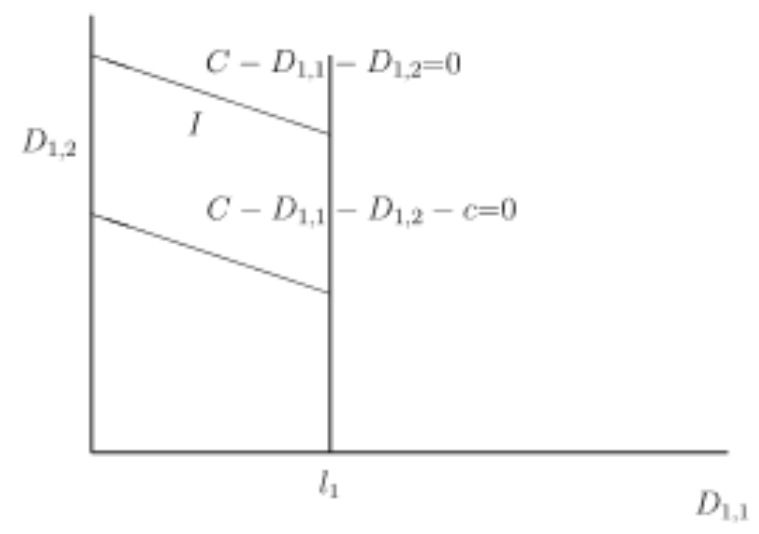

Fig. 5. Find $P\left[0<C\left(l_{1}\right) \leqslant c, D_{1,1}<l_{1}\right]$ for $c<C-l_{1}$ by integrating over region $\mathrm{I}$.

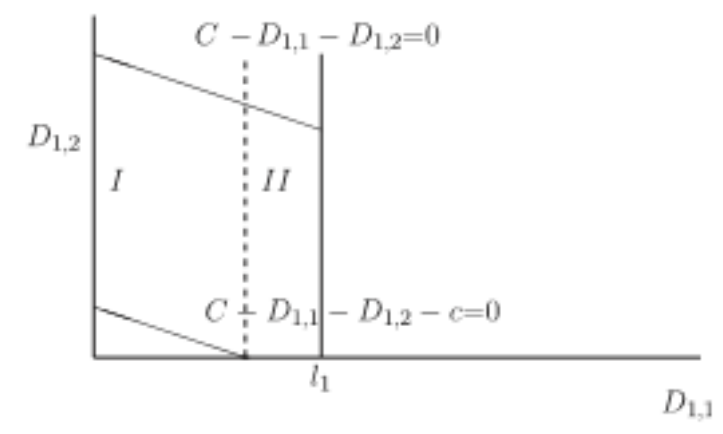

Fig. 6. Find $P\left[0<C\left(l_{1}\right) \leqslant c, D_{1,1}<l_{1}\right]$ for $c>C-l_{1}$ by integrating over regions I and II.

Case 2: $c>C-I_{1}$. From (9) and Fig. 6

(17)

$$
\begin{aligned}
P\left[C\left(l_{1}\right) \leqslant c, D_{1,1}<l_{1}, C\left(l_{1}\right)>0\right]= & \int_{0}^{C-c} \int_{C-x-c}^{C-x} f_{1,2}(y) \mathrm{d} y f_{1,1}(x) \mathrm{d} x \\
& +\int_{C-c}^{l_{1}} \int_{0}^{C-x} f_{1,2}(y) \mathrm{d} y f_{1,1}(x) \mathrm{d} x .
\end{aligned}
$$

Differentiate the above and use (9) to see that 
(18)

$g_{1}\left(c, l_{1}\right)=\left\{\int_{0}^{C-c} f_{1,2}(C-x-c) f_{1,1}(x) \mathrm{d} x\right\} / P\left[D_{1,1}<l_{1}, C\left(l_{1}\right)>0\right]$.

\section{The Two-Period Expected Return}

Suppose the booking limit for the first period is $I 1$ and that optimal booking limits $I_{2}(c)$ and $I_{2}(c$, $I_{1}$ ) for the second period are found using the procedure in Section 2.1. We will show how to write the expected return for both periods assuming $I_{1}$ is the first period booking limit. Finding the optimal $I_{1}$ then becomes a one-dimensional numerical search.

The expected revenue for the first period can be written as

$$
r_{1} E\left[\min \left\{D_{1,1}, C, l_{1}\right\}\right]+r_{2} E\left[\min \left\{\max \left\{C-l_{1}, C-D_{1,1}\right\}, D_{1,2}+d_{1}\left(D_{1,1}-l_{1}\right)^{+}\right\}\right] .
$$

The above is straightforward to calculate and explicit formulas can be found in Sen and Zhang (1999).

If demand for the cheaper fares in the first period does not reach the booking limit $I_{1}$, then no customers need wait until period 2 to obtain a cheap fare. Otherwise, a fraction $w$ of those who could not get a cheap fare will wait until the second period. The contribution to total expected revenue from the former case is given by

(20)

$$
\begin{aligned}
& P\left[D_{1,1}<l_{1}, C\left(l_{1}\right)>0\right]\left\{\int r_{1} E\left[\min \left\{D_{2,1}, c, l_{1}(c)\right\}\right] g_{1}\left(c, l_{1}\right) \mathrm{d} c\right. \\
& \left.\quad+\int r_{2} E\left[\min \left\{\max \left\{c-l_{2}(c), c-D_{2,1}\right\}, D_{2,1}+d_{2}\left(D_{2,1}-l_{2}(c)\right)\right\}\right] g_{1}\left(c, l_{1}\right) \mathrm{d} c\right\},
\end{aligned}
$$

while that from the latter is provided by 
(21)

$$
\begin{gathered}
P\left[D_{1,1} \geqslant l_{1}, C\left(l_{1}\right)>0\right] \int r_{1} E\left[\min \left\{D_{2,1}\left(l_{1}\right), c, l_{2}\left(c, l_{1}\right)\right\}\right] g_{2}\left(c, l_{1}\right) \mathrm{d} c \\
\quad+\int r_{2} E\left[\operatorname { m i n } \left\{\max \left\{c-l_{2}\left(c, l_{1}\right), c-D_{2,1}\left(l_{1}\right)\right\},\right.\right. \\
\left.\left.D_{2,1}\left(l_{1}\right)+d_{2}\left(D_{2,1}\left(l_{1}\right)-l_{2}\left(c, l_{1}\right)\right)\right\}\right] g_{2}\left(c, l_{1}\right) \mathrm{d} c .
\end{gathered}
$$

For each value of c, all of the expected values in the integrands of (20) and (21) can be explicitly calculated in a manner similar to calculating (19). Explicit formulas for $g_{1}\left(c, l_{1}\right)$ and $g_{2}\left(c, l_{1}\right)$ have been provided in Section 2.2. Thus, evaluating (20) and (21) is a straightforward numerical procedure.

The expected return for the two periods is given by adding (19), (20) and (21).

\section{Example Continued}

Continuing the example of Section 2.1.1, using the same parameters, Figs 7 and 8 display the total expected revenue as a function of the booking limit in period 1 for a series of ranges for the fractions that wait and divert. For the case where the fraction diverting in both periods is $0.1, \mathrm{Fig} .7$ displays optimal booking levels and expected revenue as a function of the fraction willing to wait, where this fraction varies from 0.4 to 0.1 . As the fraction willing to wait decreases, period 1 optimal booking limits increase from 5 to 10.

For the case where the fraction willing to wait is 0.1 , Fig. 8 displays optimal booking levels and expected revenue as a function of the fraction buying-up. For fractions willing to divert $>0.2$, the optimal booking limit in period 1 is zero.

If no strategic consumer behavior is assumed, then the sequential application of static singleperiod models, referred to as advanced static allocation (Weatherford and Bodily, 1992), would suggest a booking limit of 20. (In applying EMSR to a two-period model, the idea is to combine both periods into one, arrive at a booking limit and then revisit at period 2 when the first period demand has been observed.) Figures 7 and 8 illustrate that optimal booking limits decrease dramatically as a function of strategic behavior. The service provider is motivated to become more restrictive in its capacity allocation, initially releasing less inventory at lower prices as the level of strategic behavior increases.

Table 1 summarizes expected revenues if the airline had used EMSR type rules in the presence of strategic behavior. As the table indicates, not accounting for strategic behavior results in revenue losses in excess of $10 \%$ under certain parameter settings. 


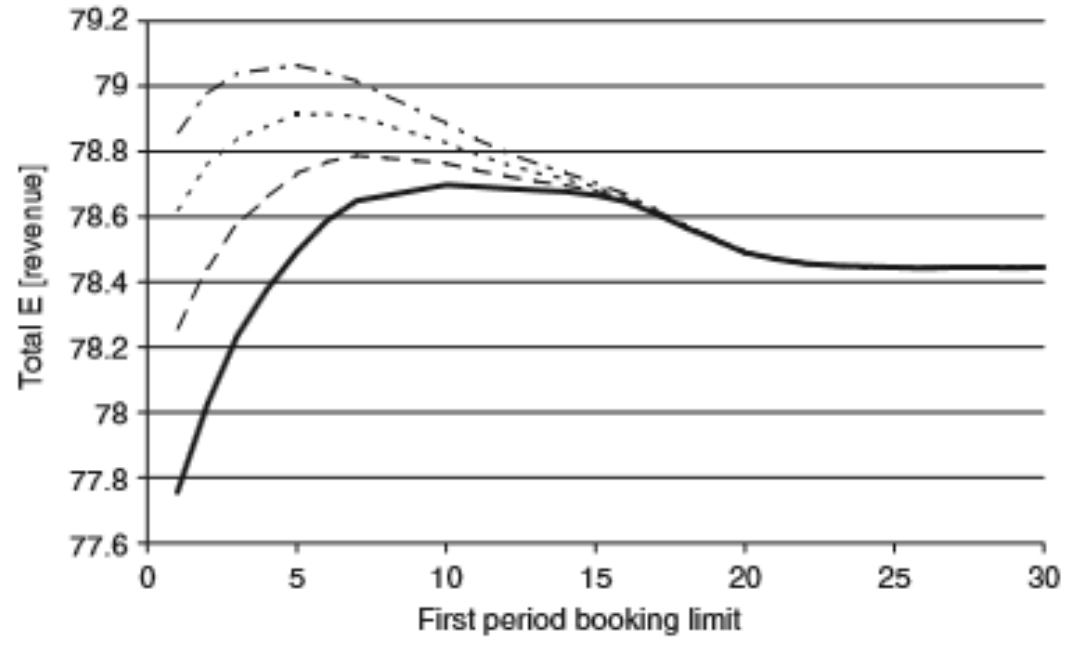

$-W=0.4 \cdots W=0.3--W=0.2-W=0.1$

Fig. 7. Total expected revenue, period 1 booking limit where fraction diverting in both periods equals 0.1 .

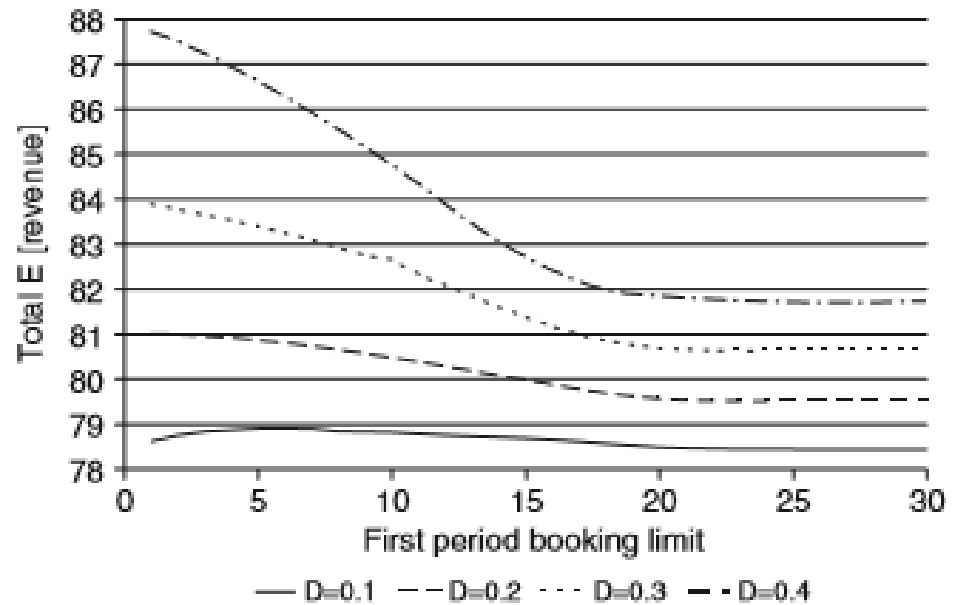

Fig. 8. Total expected revenue, period 1 booking limit where fraction waiting equals 0.1 .

Table 1

Expected revenues using EMSR versus optimal booking limits

\begin{tabular}{llllr}
\hline E[Revenues] & & & & \\
\hline \% Divert & $\%$ Wait & EMSR & Optimal & $\%$ Gain \\
\hline 10 & 10 & 78.27 & 78.70 & 0.54 \\
20 & 10 & 78.78 & 81.01 & 2.83 \\
30 & 10 & 79.09 & 83.88 & 6.06 \\
40 & 10 & 79.28 & 87.71 & 10.63 \\
10 & 10 & 78.27 & 78.70 & 0.54 \\
10 & 20 & 78.27 & 78.79 & 0.66 \\
10 & 30 & 78.27 & 78.92 & 0.82 \\
10 & 40 & 78.27 & 79.06 & 1.01 \\
\hline
\end{tabular}

EMSR, expected marginal seat revenue. 


\section{Conclusion}

Finding optimal booking limits for multi-period perishable asset revenue management models has proven to be a difficult task. Many of the multi-period models in the literature are heuristic in nature. Allowing customers to behave strategically by either diverting to another product or waiting to see whether or not a cheaper product will become available adds greatly to the modeling complexity. Anderson and Wilson (2003) showed that if customers behave strategically and firms allocate capacity via traditional approaches (independent product classes with non- strategic behavior) revenue losses to service providers can exceed $7 \%$. Customer diversion for one- period models has been investigated by a number of researchers. The contribution of this work is to demonstrate that finding optimal booking limits for a two-period model where customers may wait can be reduced to solving a number of straightforward one-dimensional problems.

\section{References}

Anderson, C., Wilson, J., 2003. Wait or buy? the strategic consumer: pricing and profit implications. Journal of the Operational Research Society 54, 299-306.

Belobaba, P., 1989. Application of probabilistic decision model to airline seat inventory control. Operations Research 37, 16.

Belobaba, P., Weatherford, L., 1996. Comparing decision rules that incorporate customer diversion in perishable asset revenue management situations. Decision Science 27, 3-43.

Bodily, S., Weatherford, L., 1995. Perishable-asset revenue management: generic and multiple-price yield management with diversion. Omega 23, 173-185.

Gerchak, Y., Parlar, M., Yee, T., 1985. Optimal rationing policies and production quantities for products with several demand classes. Canadian Journal of Administrative Science 2, 161-176.

Hersh, M., Ladany, S., 1978. Optimal seat allocation for flights with intermediate stops. Computers and Operations Research 5, 31-37.

Ladany, S., Bedi, D., 1977. Dynamic rules for flights with an intermediate stop. Omega 5, 721-730.

Lee, T., Hersh, M., 1993. A model for dynamic airline seat inventory control with multiple seat bookings. Transportation Science 27, 252-263.

Littlewood, K., 1972. Forecasting and control of passenger bookings. In AGIFORS Symposium Proceedings, Nathanya, Israel.

Pfeifer, P., 1989. The airline discount fare allocation problem. Decision Science 20, 149-157. 
Robinson, L., 1995. Optimal and approximate control policies for airline booking with sequential nonmonotonic fare classes. Operations Research 43, 2, 252-263.

Sen, A., Zhang, A., 1999. The newsboy problem with multiple demand classes. IIE Transactions 31, 431444.

Weatherford, L., Bodily, S., 1992. A taxonomy and research overview of perishable-asset revenue management: yield management, overbooking and pricing. Operations Research 40, 831-844.

Weatherford, L., Bodily, S., Pfeifer, P., 1993. Modeling the customer arrival process and comparing decision rules in perishable asset revenue management. Transportation Science 27, 12-14. 